\title{
Association between polymorphisms of fat mass and obesity-associated gene and metabolic syndrome in Kazakh adults of Xinjiang, China
}

Y.H. Hu*, J.M. Liu*, M. Zhang, R.L. Ma, H. Guo, K. Wang, J. He, Y.Z. Yan, D.S. Rui, F. Sun, L.T. Mu, Q. Niu, Y.S. Ding, J.Y. Zhang, S.G. Li and S.X. Guo

Department of Public Health and Key Laboratory of Xinjiang Endemic and Ethnic Diseases of the Ministry of Education, Shihezi University School of Medicine, Shihezi, China

*These authors contributed equally to this study.

Corresponding author: S.X. Guo

E-mail:pge888@sina.com

Genet. Mol. Res. 14 (4): 14597-14606 (2015)

Received May 27, 2015

Accepted September 11, 2015

Published November 18, 2015

DOI http://dx.doi.org/10.4238/2015.November.18.23

\begin{abstract}
The aim of this study was to assess the association between three FTO polymorphisms (rs9939609, rs8057044, and rs1421085) and metabolic syndrome (MS)-related outcomes in the low-income, rural, nomadic minority Khazakh population in far western China. A total of 489 subjects (245 MS patients, 244 controls) were included in the study and DNA samples were genotyped for the three polymorphisms by matrixassisted laser desorption/ionization time of flight mass spectrometry. The frequencies of the rs 1421085 and rs9939609 genotypes and alleles did not differ significantly between MS patients and control, while the frequencies of rs $8057044 \mathrm{G}$ alleles and GG genotypes were higher in MS patients $(P<$ 0.05 ) than in control subjects (G: 61.16 vs $53.53 \%$, GG: 39.07 vs $29.05 \%$ ) and the frequencies of rs8057044 A genotypes and alleles were lower $(P$ $<0.05$ ) in MS patients compared with controls (AA: 17.36 vs $21.99 \%$, A:
\end{abstract}


38.84 vs $46.47 \%$ ). Risk analysis of the rs 8057044 polymorphism revealed individuals with GA and GG genotypes to have 1.112 and 1.731 times higher risks of developing MS than those with the AA genotype, respectively, while the $G$ allele was found to be associated with a 1.367 times higher risk of developing MS compared with the A allele. These apparent correlations, however, did not hold true when adjusted for BMI. Weight, WC, HC, and BMI differed significantly between rs8057044 GG and AA+GA genotypes $(P<0.05)$.

Key words: FTO gene; Metabolic syndrome; Polymorphisms; Kazakh

\section{INTRODUCTION}

Metabolic syndrome (MS) is characterized by a group of metabolic disturbances, which lead to an enhanced risk of cardiovascular diseases and type 2 diabetes mellitus. Clinical characteristics include central obesity, hypertriglyceridemia, hypertension, depressed plasma highdensity lipoprotein (HDL) cholesterol, and elevated glucose (Expert Panel on Detection, Evaluation, and Treatment of High Blood Cholesterol in Adults, 2001; Eckel et al., 2005; Esposito et al., 2013). Metabolic syndrome and its concomitant complications pose a serious health problem worldwide and will gain even more importance in the future (Maury and Brichard, 2010). The prevalence of MS has increased not only in China, but also worldwide. In the USA, the prevalence of MS is estimated to be $>25 \%$ (McCullough, 2011). In Europe, various studies suggest significant geographic variation in MS prevalence and the MS prevalence in the non-diabetic population is expected to reach $23.4 \%$ in women and $25.9 \%$ in men (Qiao, 2006). In rural Australia, the prevalence of MS is very high (Janus et al., 2007). We previously showed that the MS prevalence in the Kazakh population in Xinjiang was $26.7 \%$ (Guo et al., 2011), higher than that (16.5\%) reported for adults in eight cities and provinces in China (Gu et al., 2005), indicating the unusually high prevalence of MS in the Kazakhs of Xinjiang. Epidemiological studies have reported that environmental factors such as lack of physical activity and high-fat food intake contribute to the development of MS. Nevertheless, genetic factors also play important roles in the pathogenesis of MS (Zhao et al., 2014).

In 2007 Frayling et al. (2007) reported a significant association between rs9939609 polymorphism in the fat mass and obesity associated (FTO) gene and increased risk of type 2 diabetes. The FTO gene was thus the first obesity-related locus identified. Obesity correlates strongly with MS and may therefore share a similar genetic background with MS. Indeed, the association between variants of the FTO gene and MS risk in adult populations have been researched extensively; however, the findings have been inconsistent (Wang et al., 2012; Zhou et al., 2012). China is a multi-ethnic country and in the Xinjiang Uygur Autonomous Region there are 55 ethnic groups, the Kazakh population $10 \%$ of the total population and ranked third according to the 2010 China sixth census. Because of the limited resources for public health, poor transportation, and differences in religions, cultures, lifestyles, diets, and genetic background in these ethnic groups, there have not been serious attempts to meet local public health needs. The prevention of MS has therefore become a public health issue that must be addressed in Xinjiang.

To our knowledge, few studies have been carried out to assess the relationship between the FTO gene and MS in China, and in particular the association between FTO gene factors and MS in Kazakh adults has not been investigated. The aim of this study, which was focused on Kazakh adults, was to investigate the association of the three FTO gene single nucleotide polymorphisms 
(SNPs) rs1421085, rs8057044, and rs9939609 with MS and the allele frequencies of certain SNPs in ethnic groups residing in far western China. The three abovementioned SNPs were selected based on associated studies and their potential impact on MS in Kazakh minorities.

\section{MATERIAL AND METHODS}

The Institutional Ethics Review Board at the First Affiliated Hospital of Shihezi University School of Medicine approved this study (IERB No.SHZ2010LL01). Standard university hospital guidelines including informed consent, confidentiality, voluntary participation, and anonymity were followed. All participants gave written informed consent before the study began.

\section{Study population}

This study was conducted from 2010 November to 2011 December, during which time 5692 Kazakh residents of Xinyuan County (Xinjiang Yili region) $\geq 18$ years old were selected for the study using the stratifying cluster sampling method. Xinyuan County is $\sim 3,692 \mathrm{~km}(2,294$ miles) away from Beijing, and $\sim 98 \%$ of the county's population consists of minority Muslim Kazakhs. All study subjects had resided in Xinyuan County for $>15$ years. Various biochemical parameters were assessed in all blood samples and subjects were considered to have MS based on the 2005 International Diabetes Federation definition of MS (Alberti et al., 2005). Two hundred and forty five patients with MS were randomly assigned to the case group and a total of 244 non-MS (control) subjects were randomly assigned to the control group using the group-matching sex and age method. Subjects with hypertension, diabetes, or obesity were excluded from the study.

\section{Basic data collection and MS diagnosis}

All study subjects completed a questionnaire survey on demographic information including details of disease, family, smoking and drinking habits, diet, and physical exercise. The subjects' sitting blood pressure, height, weight, waist circumference (WC), and hip circumference (HC) were also measured and recorded. Laboratory analyses of blood samples included tests for fasting triglycerides (TG), low-density lipoprotein cholesterol (LDL-C), serum total cholesterol (TC), high density lipoprotein cholesterol (HDL-C), and fasting plasma glucose (FPG), all of which were conducted using an automatic biochemical analyzer (Olympus AU400).

\section{DNA extraction}

Fasting venous blood $(200 \mu \mathrm{L})$ was taken from each study subject and a blood genomic DNA isolation kit (spin column, BioTeke, Beijing) was used to extract the whole blood genomic DNA. Extracted DNA was verified by gel electrophoresis ( $0.7 \%$ agarose). A NanoDrop spectrophotometer was used for quantitative determination of DNA concentration and purity: concentrations of $\geq 30 \mathrm{ng} /$ $\mu \mathrm{L}$ and purity levels $\left(\mathrm{OD}_{260} / \mathrm{OD}_{280}\right)$ of 1.7-2.0 were considered acceptable. Samples that met these criteria were diluted to $10-30 \mathrm{ng} / \mu \mathrm{L}$ using double-distilled water and were stored at $-80^{\circ} \mathrm{C}$.

\section{PCR amplification}

Primers were designed using the Mysequenom tool (www.mysequenom.com/Home) and (Link ok) 
Assay Designer 3.0 software. Final PCR reaction volumes were $5 \mu \mathrm{L}$, which included $0.7 \mu \mathrm{L}$ DNA, $0.1 \mu \mathrm{L}$ dNTPs, $1.35 \mu \mathrm{L}$ water, $0.5 \mu \mathrm{L}$ 10X PCR buffer, $0.4 \mu \mathrm{L} \mathrm{MgCl}, 0.15 \mu \mathrm{L}$ Taq enzymes, 1.8 $\mu \mathrm{L}$ mixture of PCR amplification primers. Cycling conditions were as follows: predenaturation at $94^{\circ} \mathrm{C}$ for $4 \mathrm{~min}$; followed by 45 cycles of denaturation at $94^{\circ} \mathrm{C}$ for $20 \mathrm{~s}$, annealing at $56^{\circ} \mathrm{C}$ for 30 $\mathrm{s}$, and extension at $72^{\circ} \mathrm{C}$ for one minute. A final extension step was carried out at $72^{\circ} \mathrm{C}$ for $3 \mathrm{~min}$, after which samples were maintained at $4^{\circ} \mathrm{C}$. Reactions were set up in an ice bath and each PCR experiment included a negative control reaction lacking DNA template.

\section{PCR product purification}

Shrimp alkaline phosphatase (SAP) was used to remove excess dNTPs from samples after PCR. This step served to ensure the accuracy of single-base extension. The final SAP reaction volumes were $2.0 \mu \mathrm{L}$, which included $0.17 \mu \mathrm{L} 10 \mathrm{X}$ SAP buffer, $1.53 \mu \mathrm{L}$ double-distilled water, and $0.3 \mu \mathrm{L} \mathrm{SAP}$ enzyme. Reactions were carried out by incubation at $37^{\circ} \mathrm{C}$ for $40 \mathrm{~min}$, followed by incubation at $85^{\circ} \mathrm{C}$ for $5 \mathrm{~min}$. The reaction products were stored at $4^{\circ} \mathrm{C}$.

\section{Single-base extension}

For single-base extension reactions, final reaction volumes were $2.0 \mu \mathrm{L}$, which included $0.619 \mu \mathrm{L}$ water, $0.2 \mu \mathrm{L} 5 \mathrm{X}$ iPlex buffer, $0.94 \mu \mathrm{L}$ primer mix, $0.2 \mu \mathrm{L}$ iPlex terminator, and $0.041 \mu \mathrm{L}$ iPlex enzyme. Reaction conditions were as follows: denaturation at $94^{\circ} \mathrm{C}$ for $30 \mathrm{~s}$; followed by 40 cycles of $94^{\circ} \mathrm{C}$ for $5 \mathrm{~s}, 52^{\circ} \mathrm{C}$ for $5 \mathrm{~s}$, and $80^{\circ} \mathrm{C}$ for $1 \mathrm{~min}$; then five cycles of incubation at $52^{\circ} \mathrm{C}$ for $5 \mathrm{~s}$ and $80^{\circ} \mathrm{C}$ for $1 \mathrm{~min}$; and finally an incubation at $72^{\circ} \mathrm{C}$ for $3 \mathrm{~min}$. Reaction products were stored at $4^{\circ} \mathrm{C}$.

\section{Matrix-assisted laser desorption-ionization time-of-flight mass spectrometry (MALDI-TOF-MS) analysis}

Single-base extension reaction products were purified using resin. A MassARRAY Nanodispenser (Sequenom, San Diego, CA, USA) was used to transfer the purification product onto a SpectroCHIP (Sequenom) chip. MALDI-TOF-MS was used for analysis. TYPER 4.0 software (Sequenom) was used to for sample analysis and classification.

\section{Statistical analysis}

Epidata 3.02 software was used to manage the study data and the double entry method was used for data entry and logical error detection. Hardy-Weinberg balance was calculated to confirm the group representation of samples and SPSS statistical software version 17.0 for Windows was used for all data analysis. Descriptive statistics were calculated to characterize the participants and study variables. The gene counting method was used to calculate the genotype and allele frequencies, chi-square testing was used for comparisons between groups, and $t$-testing was used to assess clinical variables between groups. Logistic regression analysis was used to assess risk factors, for which odds ratios (ORs) and $95 \% \mathrm{Cl}$ were calculated.

\section{RESULTS}

\section{Assessment of general data and clinical biochemical indices}

A total of 245 (male: 85; female: 160) metabolic syndrome (MS) cases and 244 (male: 83; 
female: 161) normal control (NC) subjects were included in the present study. The mean ages in the MS and NC groups were $42.51 \pm 9.61$ and $41.49 \pm 8.80$ years, respectively. The general clinical data for the subjects in each group are shown in Table 1. There was no significant difference in the gender and ages of each group $(P>0.05)$. In the MS group, the mean height, weight, BMI, WC, HC, WHR, SBP, DBP, FPG, TG, TC, and LDL-C were higher than in the control groups while mean $\mathrm{HDL}-\mathrm{C}$ was lower in the MS group than in the control group $(\mathrm{P}<0.05$ in each case).

Table 1. Comparison of clinical and biochemical indicators across to metabolic syndrome and normal control groups.

\begin{tabular}{|c|c|c|c|c|}
\hline \multirow[t]{2}{*}{ Clinical and biochemical indices } & \multirow{2}{*}{$\frac{\text { Metabolic syndrome }}{(\mathrm{MS})}$} & \multirow{2}{*}{$\frac{\text { Normal control }}{(\mathrm{NC})}$} & \multirow[t]{2}{*}{$x^{2 / t}$} & \multirow[t]{2}{*}{$P$ value } \\
\hline & & & & \\
\hline N (Male/Female) & $245(85 / 160)$ & $244(83 / 161)$ & 0.025 & 0.875 \\
\hline Age (year) & $42.51 \pm 9.61$ & $41.49 \pm 8.80$ & -1.231 & 0.219 \\
\hline Height (cm) & $163.58 \pm 8.11$ & $161.82 \pm 7.69$ & -2.461 & $0.014^{*}$ \\
\hline Weight (kg) & $74.30 \pm 13.47$ & $57.54 \pm 8.85$ & -16.260 & $<0.0001^{*}$ \\
\hline BMI $\left(\mathrm{kg} / \mathrm{m}^{2}\right)$ & $27.70 \pm 4.19$ & $21.91 \pm 2.58$ & -18.380 & $<0.0001^{*}$ \\
\hline$W C(\mathrm{~cm})$ & $93.87 \pm 9.35$ & $76.07 \pm 7.07$ & -23.736 & $<0.0001^{*}$ \\
\hline $\mathrm{HC}(\mathrm{cm})$ & $102.32 \pm 6.70$ & $91.50 \pm 5.89$ & -18.965 & $<0.0001^{*}$ \\
\hline WHR & $0.92 \pm 0.07$ & $0.83 \pm 0.05$ & -15.707 & $<0.0001^{*}$ \\
\hline $\mathrm{SBP}(\mathrm{mmHg})$ & $140.03 \pm 22.25$ & $123.40 \pm 19.30$ & -8.827 & $<0.0001^{*}$ \\
\hline $\mathrm{DBP}(\mathrm{mmHg})$ & $90.77 \pm 13.63$ & $79.37 \pm 11.41$ & -10.029 & $<0.0001^{*}$ \\
\hline $\mathrm{FPG}(\mathrm{mM})$ & $5.23 \pm 1.90$ & $4.54 \pm 0.81$ & -5.223 & $<0.0001^{*}$ \\
\hline $\mathrm{TG}(\mathrm{mM})$ & $1.76 \pm 1.57$ & $0.86 \pm 0.36$ & -8.659 & $<0.0001^{*}$ \\
\hline $\mathrm{TC}(\mathrm{mM})$ & $4.69 \pm 1.10$ & $4.30 \pm 0.95$ & -4.118 & $<0.0001^{*}$ \\
\hline HDL-C (mM) & $1.27 \pm 0.40$ & $1.57 \pm 0.41$ & 8.114 & $<0.0001^{*}$ \\
\hline LDL-C (mM) & $2.61 \pm 0.90$ & $2.16 \pm 0.61$ & -6.497 & $<0.0001^{*}$ \\
\hline
\end{tabular}

BMI, body mass index; WC, waist circumference; HC, hip circumference; WHR, waist-to-hip ratio; SBP, systolic blood pressure; DBP, diastolic blood pressure; FPG, fasting plasma glucose; TG, triglyceride; TC, total cholesterol; HDL-C, high-density lipoprotein-cholesterol; LDL-C, low-density lipoprotein-cholesterol; MS group vs Normal control group, ${ }^{*} \mathrm{P}$ $<0.05$; Data represent mean \pm SD.

\section{Hardy-Weinberg equilibrium testing of gene frequencies}

In the MS and NC groups, genotype frequencies of the rs8057044, rs9939609, and rs1421085 loci were found to adhere to the Hardy-Weinberg equilibrium (all $P>0.05$ ), indicating that three loci of the FTO gene reached genetic equilibrium and thus that the samples were indeed representative of the Kazakh population.

\section{Comparison of genotype and allele frequencies of the rs8057044, rs9939609, and rs1421085 polymorphisms across the two groups}

Analyses revealed that the distribution frequencies of the genotypes and alleles of the rs9939609 and rs1421085 FTO gene polymorphisms did not differ significantly between the MS and NC groups (all P > 0.05). The rs8057044 polymorphism, on the other hand, differed significantly between the two groups in terms of distribution frequencies of genotypes and alleles (genotypes: $X^{2}=6.227, P=0.044$; allele frequencies: $\left.X^{2}=5.748, P=0.017\right)$ : frequencies of the $r 8057044 G G$ genotypes and $\mathrm{G}$ alleles were significantly higher in MS patients than in control subject (Table 2).

\section{Risk analysis of rs 8057044 polymorphisms of the FTO gene and MS in Kazakhs}

A univariate logistic regression analysis was carried out for the rs8057044 polymorphism of the FTO gene in Kazakhs. A risk assessment was carried out with the AA genotype and the A 
allele as controls, and 95\% Cls and odds ratios (ORs) were calculated (Table 3). The risk analysis revealed that MS risk in individuals with rs8057044 GA and GG genotypes was 1.112- and 1.731fold $(95 \%$ confidence interval $(\mathrm{Cl})=1.041-2.878$; $\mathrm{P}=0.035)$ greater than in individuals with the AA genotype, and that MS risk in individuals with the $G$ allele was 1.367 -fold $(95 \%$ confidence interval $(\mathrm{Cl})=1.058-1.766, \mathrm{P}=0.017)$ greater than in individuals with the A allele. These apparent differences, however, were not significant when adjustments for BMI were made.

\begin{tabular}{|c|c|c|c|c|c|}
\hline & & Metabolic syndrome (MS) & Normal control (NC) & $x^{2}$ & $P$ \\
\hline \multirow[t]{5}{*}{ rs9939609 genotypes/allele frequencies } & $\mathrm{TT}$ & $\begin{array}{c}138 \\
(57.0)\end{array}$ & $\begin{array}{c}140 \\
(58.1)\end{array}$ & 0.306 & 0.858 \\
\hline & TA/AA & $92 / 12$ & $87 / 14$ & & \\
\hline & & $(38.0 / 5.0)$ & $(36.1 / 5.8)$ & & \\
\hline & $\mathrm{T}$ & $\begin{array}{c}368 \\
(76.0)\end{array}$ & $\begin{array}{c}367 \\
(76.1)\end{array}$ & 0.002 & 0.969 \\
\hline & A & $\begin{array}{c}116 \\
(24.0)\end{array}$ & $\begin{array}{c}115 \\
(23.9)\end{array}$ & & \\
\hline \multirow[t]{5}{*}{ rs 1421085 genotypes/allele frequencies } & $\mathrm{TT}$ & $\begin{array}{c}138 \\
(58.0)\end{array}$ & $\begin{array}{c}141 \\
(58.5)\end{array}$ & 0.399 & 0.819 \\
\hline & $\mathrm{TC} / \mathrm{CC}$ & $\begin{array}{c}88 / 12 \\
(37.0 / 5.0)\end{array}$ & $\begin{array}{c}85 / 15 \\
(35.3 / 6.2)\end{array}$ & & \\
\hline & $\mathrm{T}$ & $\begin{array}{c}364 \\
(76.5)\end{array}$ & $\begin{array}{c}367 \\
(76.1)\end{array}$ & 0.014 & 0.905 \\
\hline & $\mathrm{C}$ & 112 & 115 & & \\
\hline & & $(23.5)$ & $(23.9)$ & & \\
\hline \multirow[t]{5}{*}{ rs8057044 genotypes/allele frequencies } & GG & $\begin{array}{c}96 \\
(39.7)\end{array}$ & $\begin{array}{c}70 \\
(29.0)\end{array}$ & 6.227 & 0.044 \\
\hline & GA/AA & $104 / 42$ & $118 / 53$ & & \\
\hline & & $(43.0 / 17.3)$ & $(49.0 / 22.0)$ & & \\
\hline & G & $\begin{array}{c}296 \\
(61.2)\end{array}$ & $\begin{array}{c}258 \\
(53.5)\end{array}$ & 5.748 & 0.017 \\
\hline & A & $\begin{array}{c}188 \\
(38.8)\end{array}$ & $\begin{array}{c}224 \\
(46.5)\end{array}$ & & \\
\hline
\end{tabular}

Table 3. Risk analysis of the FTO gene polymorphism rs 8057044 and metabolic syndrome in a Kazakh population.

\begin{tabular}{|c|c|c|c|c|c|c|c|}
\hline Locus & Genotypes/allele frequencies & MS & NC & $x^{2}$ & $\mathrm{P}$ & OR & $95 \% \mathrm{Cl}$ \\
\hline \multirow[t]{5}{*}{ rs8057044 } & AA & 42 & 53 & & & 1.000 & \\
\hline & GA & 104 & 118 & 0.186 & 0.666 & 1.112 & $0.686-1.803$ \\
\hline & GG & 96 & 70 & 4.465 & 0.035 & 1.731 & $1.041-2.878$ \\
\hline & A & 188 & 224 & & & 1.000 & \\
\hline & G & 296 & 258 & 5.748 & 0.017 & 1.367 & $1.058-1.766$ \\
\hline
\end{tabular}

\section{Analyses of FTO gene polymorphisms and clinical biochemical indices in Kazakhs}

Clinical biochemical indices were compared across the different genotypes for the rs8057044, rs9939609, and rs1421085 loci of the FTO gene in Kazakhs. No significant differences were observed in the biochemical indices between the different genotypes of the rs9939609 and rs1421085 polymorphisms ( $P>0.05$; data not shown), while in the case of the rs8057044 polymorphism, significant differences $(P<0.05)$ were observed in weight, WC, HC, and BMI between the GG and AA+GA genotypes (Table 4). 


\begin{tabular}{|c|c|c|c|c|}
\hline \multirow[t]{2}{*}{ Clinical and biochemical indexes } & \multicolumn{2}{|c|}{ Genotypes } & \multirow[t]{2}{*}{$t$} & \multirow[t]{2}{*}{$\mathrm{P}$} \\
\hline & GG & AA/GA & & \\
\hline Height (cm) & $162.88 \pm 7.93$ & $162.59 \pm 7.97$ & 0.384 & 0.701 \\
\hline Weight (kg) & $67.82 \pm 15.19$ & $64.85 \pm 13.53$ & 2.194 & $0.029^{*}$ \\
\hline WC $(\mathrm{cm})$ & $86.82 \pm 12.44$ & $84.00 \pm 11.97$ & 2.428 & $0.016^{*}$ \\
\hline $\mathrm{HC}(\mathrm{cm})$ & $98.11 \pm 8.02$ & $96.29 \pm 8.44$ & 2.290 & $0.022^{*}$ \\
\hline $\mathrm{BMI}\left(\mathrm{kg} / \mathrm{m}^{2}\right)$ & $25.43 \pm 4.73$ & $24.46 \pm 4.41$ & 2.242 & $0.025^{*}$ \\
\hline FPG (mM) & $4.63 \pm 1.72$ & $4.66 \pm 1.26$ & -0.274 & 0.785 \\
\hline $\mathrm{TG}(\mathrm{mM})$ & $1.31 \pm 1.09$ & $1.30 \pm 1.28$ & 0.135 & 0.893 \\
\hline $\mathrm{TC}(\mathrm{mM})$ & $4.57 \pm 1.14$ & $4.46 \pm 1.00$ & 1.049 & 0.295 \\
\hline LDL-C (mM) & $2.45 \pm 0.97$ & $2.35 \pm 0.70$ & 1.246 & 0.213 \\
\hline HDL-C (mM) & $1.43 \pm 0.47$ & $1.42 \pm 0.41$ & 0.176 & 0.860 \\
\hline $\mathrm{SBP}(\mathrm{mmHg})$ & $132.27 \pm 21.02$ & $131.50 \pm 23.22$ & 0.361 & 0.718 \\
\hline $\mathrm{DBP}(\mathrm{mmHg})$ & $85.57 \pm 12.96$ & $84.80 \pm 14.23$ & 0.587 & 0.557 \\
\hline
\end{tabular}

\section{DISCUSSION}

In 2007, independent genome-wide association studies led to the identification of the fat mass and obesity-associated (FTO) gene (chromosome 16q12.2) as an obesity susceptibility gene (Frayling et al., 2007). A cluster of variants in the first intron of the FTO gene showed a strong association with obesity-related traits (Dina et al., 2007; Scuteri et al., 2007). Obesity is one of the major factors in MS development (Zhou et al., 2012), and although obesity and MS do not completely overlap, obesity is considered the core of MS. The FTO gene has therefore been selected as a candidate gene associated with MS (Sjögren et al., 2008). We previously reported the MS prevalence in the Kazakh population in Xinjiang to be 26.7\% (Guo et al., 2011), which is higher than the prevalence $(16.5 \%)$ reported for eight cities and provinces in China. The aim of this study was therefore to investigate the association of between the FTO SNPs rs1421085, rs8057044, and rs9939609 and MS in Kazakh adults in northwest China.

The FTO rs9939609 genotype frequency distributions in the study subjects were found to be 57.6 TT, 37.1 TA, and 5.3\% AA, while the allele distributions were $76.1 \mathrm{~T}$ and $23.9 \% \mathrm{~A}$. The FTO rs1421085 genotype frequency distributions were $58.2 \mathrm{TT}, 36.1 \mathrm{TC}$, and $5.7 \% \mathrm{CC}$, while the allele frequency distributions were $76.3 \mathrm{~T}$ and $23.7 \% \mathrm{C}$. These frequency distributions are comparable to those previously reported for Chinese populations (Xu et al., 2012; Chen et al., 2014). The overall MAFs (minor allele frequency) for FTO rs9939609 and rs1421085 gene mutation rates were found to be higher than the previously reported rates of 0.215 and 0.214 (Japanese population; Hotta et al., 2011), but lower than the reported rates of 0.42 and 0.42 (non-native American population; Wing et al., 2011). The FTO rs8057044 genotype frequency distributions in this study were 34.4 GG, 46.0 GA, and 19.7\% AA, and the allele frequency distributions were $57.3 \mathrm{G}$ and $42.7 \% \mathrm{~A}$. The overall MAF for the FTO rs8057044 gene mutation rate was lower than 0.49 (Wing et al., 2011). The frequencies of the A/C allele as well as of the AT/TC and AA/CC genotypes were lower than those reported for the European population. These discrepancies may be due to the different genetic architectures in the different populations and/or due to substantial differences in MAFs between Kazakhs and other populations.

In our study, the frequencies of the rs9939609 TT and AA genotypes and T alleles; as well as the frequencies of the rs 1421085 TT and CC genotypes and C alleles, were lower in MS patients than in control subjects; however, there was no association of these frequencies with metabolic syndrome. These findings are comparable to those previously reported for Chinese Han populations 
(Wang et al., 2010; Xu et al., 2012), but differ from others recently reported: the FTO gene rs9939609 and rs1421085 SNPs were found to be significantly associated with metabolic syndrome (Hotta et al., 2011; Zhou et al., 2012). Al-Attar et al. (2008) also reported the rs9939609 A and T allele frequencies to differ significantly between MS and control groups in a non-Caucasian multiracial study population, and furthermore reported that the A allele may be an MS risk allele. Differences between the present study findings and some of the previously reported findings may stem from different areas and ethnic genetic factors affecting the distributions of FTO polymorphisms.

In this study, the frequencies of the rs8057044 GG genotypes and G alleles in MS patients were shown to be significantly higher than those in the control subjects. A significant association between rs8057044 and MS was initially demonstrated for the Kazakh population [per $\mathrm{G}$ allele odds ratio $(\mathrm{OR})=1.367,95 \%$ confidence interval $(\mathrm{Cl})=1.058-1.766, \mathrm{P}=0.017$ ] and risk assessment deemed the $\mathrm{G}$ allele a risk factor for MS; however, these differences were no longer statistically significant after adjustments were made for BMI. These findings suggest that this apparent association is mediated via BMI (González-Sánchez et al., 2009; Xi et al., 2010; Zhao et al., 2014). Variations in the FTO gene have previously been attributed to overall lipid effects of obesity-related metabolic traits in Japan (Hotta et al., 2011), China, and Taiwan's (Chang et al., 2008) yellow race populations.

In this study, the relationship between the 3 loci polymorphisms of the FTO gene and clinical biochemical indicators of MS were investigated. The study results suggest that there is no significant difference among the two polymorphisms rs9939609 and rs1421085 in terms of clinical and biochemical indices, a finding that is in agreement with reports on Japanese and Chinese populations (Horikoshi et al., 2007; Li et al., 2008). In the case of the rs8057044 polymorphism, however, various indices (weight, WC, HC, and BMI) did differ significantly across the different genotypes: subjects with the AA/GA genotype were found to have lower weight, WC, $\mathrm{HC}$, and $\mathrm{BMI}$ than those with the GG genotypes. These findings suggest that the AA/GA genotype is associated with lower body weight, WC, $\mathrm{HC}$, and $\mathrm{BMI}$ and may therefore reduce the risk of obesity, playing a beneficial effect in the development of MS.

\section{CONCLUSIONS}

Based on the study results discussed above, FTO rs8057044 polymorphism may be associated with the occurrence of metabolic syndrome, and specifically the $G$ allele may be considered a risk factor for MS, an association possibly mediated through BMI. Nevertheless, no significant associations of the FTO rs9939609 and rs1421085 polymorphisms with the occurrence of metabolic syndrome were observed. Local and global studies on FTO polymorphism and MS have yielded contradicting results, which may arise from ethnic and regional differences affecting the influence of rs8057044 polymorphism on metabolic parameters in patients with MS.

This is the first report of an association between the rs9939609, rs1421085, and rs8057044 FTO gene polymorphisms with MS in Kazakh adults in China. The etiological mechanism of MS is complex and apart from the effects of the patients' regions, racial differences, and other environmental factors, synergies or interfering actions of other known or unknown genes cannot be ruled out. In addition to China, Kazakh people are also widely distributed in several countries including the Soviet Republic of Kazakhstan, Uzbekistan, Turkmenistan, Kyrgyzstan, Tajikistan, the People's Republic of Mongolia, and Afghanistan. This study therefore not only assessed the association between FTO polymorphisms and the incidence of MS in low-income, rural, and nomadic Kazakh minority people in China's far west but also explored the possible pathogenesis 
of MS from the perspective of genetics, which may provide a new way of thinking for the diagnosis, prevention, and treatment of MS at the genetic level in the Kazakh population.

\section{Conflicts of interest}

The authors declare no conflict of interest.

\section{ACKNOWLEDGMENTS}

Research supported by grants from the National Science and Technology Support Projects for the "Eleventh Five-Year Plan" of China (\#2009BAl82B04) and was partly supported by a grant from the Department of Public Health and the Key Laboratory of Xinjiang Endemic and Ethnic Diseases of the Ministry of Education, Shihezi University School of Medicine.

\section{REFERENCES}

Al-Attar SA, Pollex RL, Ban MR, Young TK, et al. (2008). Association between the FTO rs9939609 polymorphism and the metabolic syndrome in a non Caucasian multi-ethnic sample. Cardiovasc. Diabetol. 7: 5.

Alberti KG, Zimmet P and Shaw J (2005). IDF Epidemiology Task Force Consensus Group. The metabolic syndrome-a new worldwide definition. Lancet 366: 1059-1062.

Chang YC, Liu PH, Lee WJ, Chang TJ, et al. (2008). Common variation in the fat mass and obesity-associated (FTO) gene confers risk of obesity and modulates BMI in the Chinese population. Diabetes. 57: 2245-2252.

Chen H, Wang KN, Tu LX, et al. (2014). FTO polymorphisms associated with metabolic components in Chinese obese children. China Mod. Doctor 52: 24-27.

Dina C, Meyre D, Gallina S, Durand E, et al. (2007). Variation in FTO contributes to childhood obesity and severe adult obesity. Nat. Genet. 39: 724-726.

Eckel RH, Grundy SM and Zimmet PZ (2005). The metabolic syndrome. Lancet 365: 1415-1428.

Esposito K, Chiodini P, Capuano A, Bellastella G, et al. (2013). Colorectal cancer association with metabolic syndrome and its components: a systematic review with meta-analysis. Endocrine 44: 634-647.

Expert Panel on Detection, Evaluation, and Treatment of High Blood Cholesterol in Adults (2001). Executive Summary of The Third Report of The National Cholesterol Education, Evaluation, and Treatment of High Blood Cholesterol In Adults (Adult Treatment Panel III). JAMA 285: 2486-2497.

Frayling TM, Timpson NJ, Weedon MN, Zeggini E, et al. (2007). A common variant in the FTO gene is associated with body mass index and predisposes to childhood and adult obesity. Science 316: 889-894.

González-Sánchez JL, Zabena C, Martínez-Larrad MT, Martínez-Calatrava MJ, et al. (2009). Variant rs9939609 in the FTO gene is associated with obesity in an adult population from Spain. Clin. Endocrinol. (Oxf). 70: 390-393.

Gu D, Reynolds K, Wu X, Chen J, et al. (2005). Prevalence of the metabolic syndrome and overweight among adults in China. Lancet 365: 1398-1405.

Guo H, Ma RL, Zhang JY, et al. (2011). Comparative analysis of epidemic characteristic of metabolic syndrome of Kazakh and Hans in Xinjiang. Chin. J. Hyperten. 19: 538-543.

Horikoshi M, Hara K, Ito C, Shojima N, et al. (2007). Variation in the HHEX gene are associated with increased risk of type 2 diabetes in the Japanese population. Diabetologia 50: 2461-2466.

Hotta K, Kitamoto T, Kitamoto A, Mizusawa S, et al. (2011). Association of variations in the FTO, SCG3 and MTMR9 genes with metabolic syndrome in a Japanese population. J. Hum. Genet. 56: 647-651.

Janus ED, Laatikainen T, Dunbar JA, Kilkkinen A, et al. (2007). Overweight, obesity and metabolic syndrome in rural southeastern Australia. Med. J. Aust. 187: 147-152.

$\mathrm{Li} \mathrm{H}, \mathrm{Wu}$ Y and Loos RJ (2008). Variants in the fat mass and obesity-association (FTO) gene are not associated with obesity in a Chinese Han population. Diabetes 57: 264-268.

Maury E and Brichard SM (2010). Adipokine dysregulation, adipose tissue inflammation and metabolic syndrome. Mol. Cell Endocrinol. 314: 1-16.

McCullough AJ (2011). Epidemiology of the metabolic syndrome in the USA. J. Dig. Dis. 12: 333-340.

Qiao Q (2006). Comparison of different definitions of the metabolic syndrome in relation to cardiovascular mortality in European men and women. Diabetologia 49: 2837-2846. 
Scuteri A, Sanna S, Chen WM, Uda M, et al. (2007). Genome-wide association scan shows genetic variants in the FTO gene are associated with obesity-related traits. PloS Genet. 3: e115.

Sjögren M, Lyssenko V, Jonsson A, Berglund G, et al. (2008). The search for putative unifying genetic factors for components of the metabolic syndrome. Diabetologia 51: 2242-51.

Wang H, Dong S, Xu H and Yang J (2012). Genetic variants in FTO associated with metabolic syndrome: a meta- and genebased analysis. Mol. Biol. Rep. 39: 5691-5698.

Wang T, Huang Y, Xiao XH, Wang DM, et al. (2010). The association between common genetic variation in the FTO gene and metabolic syndrome in Han Chinese. Chin. Med. J. 123: 1852-1858.

Wing MR, Ziegler JM, Langefeld CD, Roh BH, et al. (2011). Analysis of FTO gene variants with obesity and glucose homeostasis measures in the multiethnic Insulin Resistance Atherosclerosis Study cohort. Int. J. Obes. (Lond) 35: 1173-1182.

Xi B, Shen Y, Zhang M, Liu X, et al. (2010). The common rs9939609 variant of the fat mass and obesity-associated gene is associated with obesity risk in children and adolescents of Beijing, China. BMC Med. Genet. 11: 107.

Xu ZY, Song JY, Wang HJ and Li G (2012). Study on the relationship between MC4R, FTO gene polymorphisms and metabolic syndrome-related phenotypes. Mod. Prev. Med. 39: 1866-1870.

Zhao X, Xi B, Shen Y, Wu L, et al. (2014). An obesity genetic risk score is associated with metabolic syndrome in Chinese children. Gene 535: 299-302.

Zhou D, Liu H, Zhou M, Wang S, et al. (2012). Common variant (rs9939609) in the FTO gene is associated with metabolic syndrome. Mol. Biol. Rep. 39: 6555-6561. 